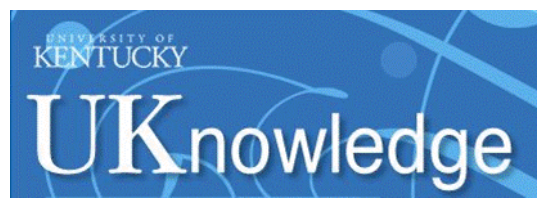

University of Kentucky

UKnowledge

\title{
Lisinopril or Coreg CR in Reducing Cardiotoxicity in Women with Breast Cancer Receiving Trastuzumab: A Rationale and Design of a Randomized Clinical Trial
}

\author{
Maya Guglin \\ University of Kentucky, maya.guglin@uky.edu \\ Pamela Munster \\ University of San Francisco \\ Angelina Fink \\ University of South Florida \\ Jeffrey Krischer \\ University of South Florida
}

Follow this and additional works at: https://uknowledge.uky.edu/internalmedicine_facpub

Part of the Cardiology Commons, Clinical Trials Commons, and the Oncology Commons

Right click to open a feedback form in a new tab to let us know how this document benefits you.

\section{Repository Citation}

Guglin, Maya; Munster, Pamela; Fink, Angelina; and Krischer, Jeffrey, "Lisinopril or Coreg CR in Reducing Cardiotoxicity in Women with Breast Cancer Receiving Trastuzumab: A Rationale and Design of a Randomized Clinical Trial" (2017). Internal Medicine Faculty Publications. 175.

https://uknowledge.uky.edu/internalmedicine_facpub/175

This Article is brought to you for free and open access by the Internal Medicine at UKnowledge. It has been accepted for inclusion in Internal Medicine Faculty Publications by an authorized administrator of UKnowledge. For more information, please contact UKnowledge@lsv.uky.edu. 


\section{Lisinopril or Coreg CR in Reducing Cardiotoxicity in Women with Breast Cancer Receiving Trastuzumab: A Rationale and Design of a Randomized Clinical Trial}

Digital Object Identifier (DOI)

https://doi.org/10.1016/j.ahj.2017.03.010

Notes/Citation Information

Published in American Heart Journal, v. 188, p. 87-92.

(c) 2017 Elsevier Inc. All rights reserved.

This manuscript version is made available under the CC-BY-NC-ND 4.0 license

https://creativecommons.org/licenses/by-nc-nd/4.0/.

The document available for download is the author's post-peer-review final draft of the article. 


\title{
Lisinopril or Coreg CR in Reducing Cardiotoxicity in Women with Breast Cancer Receiving Trastuzumab: a Rationale and Design of a Randomized Clinical Trial
}

\author{
Maya Guglin ${ }^{1}$, Pamela Munster ${ }^{2}$, Angelina Fink ${ }^{3}$, and Jeffrey Krischer ${ }^{3}$ \\ 1 University of Kentucky, Lexington, KY \\ 2University of San Francisco, San Francisco, CA \\ 3University of South Florida, Tampa, FL
}

\begin{abstract}
Background-Trastuzumab (TZB) is an established therapy for HER2 positive breast cancer. The use of TZB is commonly associated with cardiotoxicity manifesting as asymptomatic decrease in left ventricular ejection fraction (LVEF) or overt heart failure. Several studies demonstrated favorable effects of angiotensin converting enzyme (ACE) inhibitors and beta blockers (BB) in the prevention of chemotherapy-induced cardiotoxicity. We hypothesize that patients, randomized to receive an ACE inhibitor or a beta-blocker during trastuzumab therapy for breast cancer, will maintain a higher LVEF than patients randomized to placebo.
\end{abstract}

Methods and Results-We designed a prospective, multicenter, randomized, phase II placebocontrolled clinical trial to evaluate the effects of an ACE inhibitor (lisinopril) and a $\beta$-blocker (carvedilol phosphate-extended release) on cardiotoxicity in patients with breast cancer who are receiving adjuvant or neoadjuvant TZB therapy. The primary objectives include 1) comparison of incidence of cardiotoxicity and 2) comparison of LVEF as a continuous variable in between the arms. Cardiotoxicity was defined as an absolute decrease in LVEF from baseline of $\geq 10 \%$ at follow-up or an absolute decrease of $\geq 5 \%$ in LVEF from baseline for individuals with $<50 \%$ LVEF at follow-up.

The target accrual is 468 participants, representing patients both with and without anthracycline exposure. The enrollment is completed. The trial is co-sponsored by University of South Florida and National Cancer Institute. The LVEF is being evaluated by echocardiography or multigated acquisition scan.

Corresponding author: Maya Guglin, MD, PhD, 900S Limestone Street, Lexington, KY, 40507, 248-346-3507, Fax 859-355-9603, Maya.guglin@uky.edu.

Disclosures: None.

Publisher's Disclaimer: This is a PDF file of an unedited manuscript that has been accepted for publication. As a service to our customers we are providing this early version of the manuscript. The manuscript will undergo copyediting, typesetting, and review of the resulting proof before it is published in its final citable form. Please note that during the production process errors may be discovered which could affect the content, and all legal disclaimers that apply to the journal pertain. 
Conclusion-If we can demonstrate that the use of an ACE inhibitor or a BB can reduce the degree of TZB-induced cardiotoxicity it is hoped that patients will receive complete and uninterrupted TZB therapy for breast cancer without compromising cardiac function.

Clinical trial registration-SCUSF 0806 NCT01009918 (url https://clinicaltrials.gov/ct2/show/ NCT01009918).

\section{Keywords}

heart failure; chemotherapy-induced cardiotoxicity; cardio-oncology

\section{Introduction}

Human Epidermal Growth Factor receptor type 2 (HER2) is a type of transmembrane tyrosine kinase receptor that normally regulates cell growth and survival, as well as adhesion, migration, differentiation, and other cellular responses. It is overexpressed in 20$30 \%$ of invasive breast carcinomas. HER2 positivity conveys a poor prognosis and decreased overall survival as well as an altered response to anthracycline chemotherapeutic regimens. Trastuzumab (Herceptin; Genentech, Inc.) is a recombinant humanized monoclonal antibody that binds to the extracellular domain of the HER2 receptor. Use of trastuzumab (TZB) in HER2 positive breast cancer became the greatest breakthrough therapy which reduces mortality and dramatically improves disease-free survival. Pooled results from five randomized trials of adjuvant trastuzumab showed a significant reduction of mortality $(\mathrm{p}<$ $0.00001)$, recurrence $(p<0.00001)$, metastases rates $(p<0.00001)$ and second tumors other than breast cancer $(\mathrm{p}=0.007)$ as compared to no adjuvant TZB patients ${ }^{1}$.

In 2001, Slamon et al reported 8.8 to $11 \%$ incidence of cardiotoxicity in patients receiving paclitaxel and trastuzumab comparing to 1 to $4 \%$ in those who received paclitaxel alone, and 26 to $28 \%$ in those on trastuzumab and anthracyclines (AC) versus 6 to $9.6 \%$ on $\mathrm{AC}$ alone ${ }^{2}$. Multiple studies have been done since and have shown similar results ${ }^{3-5}$. Most of the cases of trastuzumab-induced cardiomyopathy are reversible, however, once trastuzumab is discontinued, oncologists are frequently hesitant to re-expose patients to this agent. Cardiotoxicity results in discontinuation of trastuzumab in up to $19 \%$ of patients ${ }^{3}$. In our retrospective study, $19.5 \%$ of patients permanently or temporarily discontinued TZB secondary to cardiomyopathy ${ }^{6}$.

There are some reports, primarily from single center studies, demonstrating the protective effects of angiotensine converting enzyme (ACE) inhibitors and beta blockers (BB) in chemotherapy-induced cardiotoxicity. Specifically, Cardinale et al. ${ }^{7}$ reported a $100 \%$ prevention of AC-induced cardiomyopathy by enalapril, an ACE inhibitor. Similarly, Kalay et al. ${ }^{8}$ described a highly successful use of carvedilol, a BB, for prevention of chemotherapy-associated cardiac toxicity. Based on these reports, as well as on our preliminary data ${ }^{6}$, we hypothesized that patients randomized to receive an ACE inhibitor or a BB, simultaneously with initiation of TZB for HER2 positive breast cancer, would preserve better cardiac function than those randomized to placebo. We defined cardiotoxicity as decrease in LVEF of $<50 \%$ with a $5 \%$ drop from baseline, or a $>10 \%$ decrease in ejection fraction after initiating trastuzumab. 


\section{Methods}

We designed a prospective, multicenter, randomized, phase II placebo-controlled clinical trial evaluating the effects of an ACE inhibitor (lisinopril) and a $\beta$-blocker (carvedilol phosphate-extended release) on cardiotoxicity in patients with breast cancer who are receiving adjuvant or neoadjuvant TZB therapy. The primary objective of this study is to determine if administration of lisinopril or carvedilol phosphate-ER, compared to placebo, will reduce the incidence of trastuzumab-induced cardiotoxicity, as measured by LVEF, in patients receiving adjuvant or neoadjuvant therapy for HER2 positive breast cancer. The secondary objectives of this study are to: 1) determine whether participants on active agent have fewer interruptions in TZB therapy due to cardiomyopathy, determine if treatment effects are consistent in anthracycline and non-anthracycline cohorts, evaluate changes in quality of life among the treatment groups during the study intervention, evaluate the long term effects of the prevention of cardiomyopathy and impact on HRQL for either or both active agents, and to compare the predictive value of Troponin I and BNP in the identification of TZB cardiotoxicity.

We chose the extended release Coreg XR, rather than generic carvedilol, because, like lisinopril, it is taken only once daily, and carvedilol has to be taken twice a day. A double blind design of the study mandated a matching pattern of use of all the drugs in the trial.

The trial is registered as SCUSF 0806 (SunCoast University of South Florida) and has a ClinicalTrials.gov Identifier NCT01009918. The study described was supported by Award Number U10CA081920 from the National Cancer Institute. Because the trial was designed as a "real life" study, we allowed the participating centers to select their preferred imaging modality. The LVEF is being evaluated by echocardiography or multigated acquisition scan, depending on the preferences of the clinical investigators. To provide consistency within centers, we required utilization of similar imaging modality throughout the follow-up, i.e. if the patient had an echocardiogram at the baseline, subsequent images were also obtained by echocardiography. Because we realize that multitude of the sites may provide inconsisted readings, the Echo substudy was designed, where the analysis of sample echocardiograms is being done by Duke core echocardiographic laboratory. Specifically, echocardiograms of all patients meeting the definition of cardiotoxicity per their local sites, as well as their agematched controls without cardiotoxicity by local readers, were sent to the core laboratory. The results will be reported separately from the main trial.

\section{Definition of TZB-induced Cardiotoxicity}

TZB-induced cardiotoxicity usually manifests in decrease of left ventricular ejection fraction (LVEF). There is no uniform definition of TZB -induced cardiotoxicity. When our trial was being designed, the literature demonstrated high heterogeneity in the definitions ${ }^{9}$. Examples are shown in Table 1.

For SCUSF 0806 trial, we chose the definition of cardiotoxicity similar to Piccart-Gebhart et al. ${ }^{10}$ :

1. A decrease in LVEF of $\geq 10 \%$ in patients whose LVEF is $\geq 50 \%$ or 
2. A drop in LVEF of at least $5 \%$ from baseline in patients whose LVEF decreases to less than $50 \%$.

Baseline LVEF must be measured within 6 weeks of study entry.

\section{Subjects and Randomization}

Subjects meeting the inclusion/exclusion criteria and giving informed consent are randomized in a 1:1:1 ratio into the two treatment and control groups (lisinopril 10mg a day versus Coreg XR 10mg versus placebo). The target enrollment is 468 patients at 128 centers. The enrollment is completed. Half of the patients were exposed to anthracyclines prior to trastuzumab therapy, while the other half was not. In terms of randomization, once a patient consented to study, the site would call a toll free number and place the participant eligibility out sources information into the automated system that would simultaneously enroll and randomize the patient to study agent. The site pharmacy would receive and automated email with a bottle number to dispense with the patients assigned blinded study agent.

\section{Inclusion Criteria}

1.1 Women $\geq 18$ years of age

1.2 HER2 positive breast cancer

1.3 Scheduled to receive adjuvant trastuzumab therapy

1.4 LVEF $\geq 50 \%$ by MUGA scan or echocardiogram, based on institutional/ clinician preference

1.5 Normal renal and hepatic function

1.6 Systolic blood pressure of $>90 \mathrm{~mm} \mathrm{Hg}$ (sitting)

1.7 Pulse $\geq 60$ beats/minutes

1.8 Able and willing to give informed consent

1.9 Signed HIPAA compliant research authorization to release Personal Health Information to the SunCoast Research Base.

\section{Exclusion criteria}

1.10 Prior treatment with trastuzumab

1.11 Current treatment with ACE inhibitors, BB or digoxin

1.12 Known cardiac history: heart failure, myocardial infarction, radiation-induced cardiac dysfunction, clinically significant arrthymias, etc.

1.13 Known contraindication to either ACE inhibitors or BB

1.14 History of bronchial asthma or related bronchospastic conditions

1.15 Hereditary or idiopathic angioedema

1.16 Pregnant or breast feeding

1.17 Metastatic disease 


\section{Study Procedures and Follow-up}

The study flow is summarized in Figure 1. Trastuzumab therapy was interrupted when the subjects met the definition of cardiotoxicity. If the patient developed heart failure during the study, trastuzumab was discontinued, study assignment unblinded, and further care provided by local cardiologist in accordance with the guidelines. Nevertheless, follow-up visits take place every three months, with documentation of the disease status, medical management, LVEF, BNP \& Troponin I; as well as QOL questionnaire at month 6 and 12.

\section{Statistical Analyses}

The primary aim of this study is to determine if treatment with lisinopril or carvedilol phosphate ER results in reduction in incidence of trastuzumab-induced cardiotoxicity after 52 weeks of treatment as measured by preservation of LVEF, and comparison of the LVEF of each treatment group with the placebo arm (where LVEF is a continuous variable). The study is stratified according to whether patients received an anthracycline-containing regimen or not to ensure balanced randomization.

The study employs a two stage analysis plan. We will first compare each active agent arm with the placebo arm. If a reduction effect is statistically significant in both agents (ACE and $\mathrm{BB}$ ), we will examine if there is any difference between the two study agents, recognizing we do not have the same statistical power for this secondary analysis. If one or neither of the active agent arms is statistically significantly different from the placebo arm, then the secondary stage analysis will not be necessary. Kaplan-Meier survival curves will be constructed to reflect time until event rates and the logrank statistic will be used to compare them. Secondary end points will be addressed by using a Student-t test to compare the changes of LVEF as a continuous measure between the two arms (treatment arms with placebo) and a Pearson chi-square test will be used to compare the proportions of decreased LVEF in each arm. For comparisons of each treatment with placebo, we will consider onesided 0.025 significant levels to adjust for the multiple testing. A mixed linear model will be utilized to analyze the pattern of LVEF change during the course of this study using the data from all follow-up visits. Furthermore, a proportional hazards model will be used to control for potential confounders such as use of other cardiotoxic agents (i.e., AC).

Quality of life changes will be evaluated using the European Organization for research and Treatment of Cancer Core Quality of Life Questionnaire (EORTC QLQ)-C30 V 3.0 at baseline and at the end of treatment (end of trastuzumab therapy or week 52). The analysis plan will utilize a linear regression model to consider the treatment effects on these measurements (dependent variables) adjusted for age and other covariates to compare the treatment arms. The observed data will be evaluated for normality and appropriate transformation introduced prior to conducting the analysis. To assess the long term effect of the study agents, follow-up measurements will be collected at study months 18 and 24 (or 6 and 12 months after the last dose of trastuzumab, if it was discontinued before week 52). This exploratory analysis will be completed to determine the long term effects on the prevention of cardiomyopathy and impact on QOL for either or both study agents. The treatment groups will be compared to using appropriate two sample tests. 
Sample Size-The sample size calculation is based on the comparisons of each treatment with placebo. Using a conservative incidence estimate, we hypothesize that $15 \%$ of women treated for breast cancer with adjuvant trastuzumab will have decreased LVEF in the placebo arm as compared to $5 \%$ of the women on the treatment arms. To detect a $10 \%$ difference in women who exhibit a decrease in LVEF by the additional medication of lisinopril or carvedilol, a sample size of 141 participants per arm is required to achieve $80 \%$ power for a one sided test at the 0.025 significance level. Assuming a 10\% drop-out rate, 468 subjects were randomized to one of the three arms.

As possible, all statistical analyses will be based on the intent-to-treat principle. Subjects will be analyzed according to their randomized assignment.

Biomarkers: B-type natriuretic peptide and Troponin-B-type natriuretic peptide (BNP) and Troponin are the biomarkers being checked regularly while participating on study. The will be used in an effort try to identify patients at risk for cardiomyopathy before they develop symptoms and before the drop of LVEF can be appreciated by echocardiography or MUGA. Cardiac troponin I (TnI) may be a very reliable biomarker. In a single center study by Cardinale et al. ${ }^{7}$, only $5 \%$ of patients without TnI elevation in the course of treatment developed cardiomyopathy. On the other hand, $62 \%$ of those with elevated TnI later had reduced EF. Moreover, there was less recovery of cardiac function in those patients who had elevated TnI. If this is confirmed in a randomized clinical trial like the one we are doing now, the strategy may be changed from imaging patients every three months which is cumbersome and costly to screening them with TnI first, and selectively imaging only those in whom it is abnormal. There is no data on head to head comparison on BNP and TnI. Receiver operating characteristic (ROC) curves will be used to determine the value of these biomarkers in predicting trastuzumab-induced cardiotoxicity: The null hypothesis is that areas under ROC (AUCROC) for the two biomarkers regarding identification of trastuzumab induced cardiotoxicity (primary endpoint) are equal. The AUCROC will be tested using Delong's nonparametric approach, applying generalized Ustatistics to generate an estimated covariance matrix for correlated ROC curves. Due to the uncertainty about the treatment effect of the active drugs, this analysis will be limited to the placebo group only. With approximate $15 \%$ incidence rate in the placebo group, the proposed sample size will achieve a power greater than $80 \%$ to reject the null if the true

Safety management-Patients are monitored for adverse events attributable to study agent at every study visit according to the Common Terminology Criteria for Adverse Events (CTCAE) v4.0. All adverse events attributable to study agent were reported to the SunCoast CCOP Research Base staff according to FDA guidelines. The study was also reviewed for safety and progress by a Data Safety Monitoring Board every 6 months.

\section{Discussion}

This prospective randomized multicenter double blind placebo-controlled trial is the largest study to address the prevention of TZB-induced cardiotoxicity. Unlike this study, most similar studies addressing this important clinical issue are conducted in single centers, with smaller sample size and less rigorous design 7,8 . 
The incidence of cardiomyopathy reported in individual clinical trials varies widely and ranges from $7 \%{ }^{4}$ to $34 \%{ }^{11}$. In an overview of selected clinical trials on adjuvant breast cancer treatment, Ewer et al. ${ }^{12}$ reported that overt clinical heart failure developed in $<4 \%$ of patients, while asymptomatic decline in LVEF by 10 points was seen in $\leq 30 \%$ of patients. Jones et al. report adverse cardiac effects in 5 of 28(17.9\%), with two of them (3.8\%) symptomatic ${ }^{13}$. Bhimaraj et al. ${ }^{14}$ report cardiac dysfunction in $32 \%$, including $14.3 \%$ symptomatic, in their mixed adjuvant and metastatic patients. The University of Texas M.D. Anderson Cancer Center (Houston, TX), observed cardiomyopathy in $28 \%$ of patients, with a more stringent definition of $\mathrm{EF}$ decline; $\geq 20 \%$ from the baseline. There was also one cardiac-related death $(0.5 \%)^{15}$. Our sample size calculation, based on the anticipated cardiotoxicity rate of $15 \%$, appears to be quite realistic.

In terms of clinical course of cardiac dysfunction which is secondary to TZB, it appears to be mostly reversible. The MD Anderson Cancer center study, in which patients were referred for suspected TZB-related cardiomyopathy, LVEF decreased from $61 \%+/-13 \%$ at baseline to $43 \%+/-16 \%$ ( $<$ < 0.0001$)$ after trastuzumab, and increased to $56 \%+/-11 \%$ after the discontinuation of therapy 16 .

Improvement in LVEF occurs after discontinuation of TZB, with cardiac medications or without them, and re-exposure to TZB usually does not result in further decline in $\operatorname{LVEF}^{4}, 15,17$. However, current protocols mandate discontinuation of TZB after LVEF becomes abnormally low $(<50 \%)$, and many women with breast cancer experience interruptions in therapy ${ }^{6}$. We designed our trial in an effort to prevent the development of TZB-related cardiac dysfunction, using the medications that were reported to provide some favorable effects, in a randomized, double blinded, placebo controlled population. Our ultimate goal is to find the way to protect the heart and to avoid interruptions in the course of TZB.

The trial is designed for community oncology groups rather than large institutions.

While the trial was in progress, results of two other randomized trials were published. Although on a smaller scale, the two groups of investigators tried to answer similar question.

In the Multidisciplinary Approach to Novel Therapies in Cardio-Oncology Research (MANTICORE 101-Breast) trial, with a double-blinded, placebo-controlled design, patients with HER2-positive breast cancer were randomly assigned to receive treatment with perindopril, bisoprolol, or placebo (1:1:1, with 33,31 , and 30 patients, respectively) for the duration of trastuzumab adjuvant therapy. After 17 cycles of trastuzumab, indexed left ventricular end diastolic volume increased similarly in all patients, but trastuzumab-induced decline in LVEF was attenuated in bisoprolol-treated patients $(-1 \pm 5 \%)$ relative to the perindopril $(-3 \pm 4 \%)$ and placebo $(-5 \pm 5 \%)$ groups $(\mathrm{P}=.001)$. Perindopril and bisoprolol use were independent predictors of maintained LVEF on multivariable analysis 18 .

The second trial utilized angiotensin II antagonist candesartan for prevention of trastuzumab related cardiotoxicity (defined as a decline in left ventricular ejection fraction (LVEF) of more than $15 \%$ or a decrease below the absolute value $45 \%$ ). in adjuvant setting. With a total of 206 participants, randomized 1:1 into candesartan or placebo, the trial demonstrated no 
diffirence in the number of cardiac events between the groups. Moreover, candesartan did not affect changes in NT-proBNP and troponin values ${ }^{19}$.

The controversial results of these two studies underscores the timeliness of our trial.

\section{Acknowledgments}

Funding sources: The study described was supported by Award Number U10CA081920 from the National Cancer Institute.

The authors are solely responsible for the design and conduct of this study, all study analyses, the drafting and editing of the paper and its final contents.

\section{References}

1. Viani GA, Afonso SL, Stefano EJ, De Fendi LI, Soares FV. Adjuvant trastuzumab in the treatment of her-2-positive early breast cancer: a meta-analysis of published randomized trials. BMC cancer. 2007; 7:153. [PubMed: 17686164]

2. Slamon DJ, Leyland-Jones B, Shak S, Fuchs H, Paton V, Bajamonde A, et al. Use of chemotherapy plus a monoclonal antibody against HER2 for metastatic breast cancer that overexpresses HER2. The New England journal of medicine. 2001; 344(11):783-92. [PubMed: 11248153]

3. Romond EH, Perez EA, Bryant J, Suman VJ, Geyer CE Jr, Davidson NE, et al. Trastuzumab plus adjuvant chemotherapy for operable HER2-positive breast cancer. The New England journal of medicine. 2005; 353(16):1673-84. [PubMed: 16236738]

4. Tripathy D, Seidman A, Keefe D, Hudis C, Paton V, Lieberman G. Effect of cardiac dysfunction on treatment outcomes in women receiving trastuzumab for HER2-overexpressing metastatic breast cancer. Clinical breast cancer. 2004; 5(4):293-8. [PubMed: 15507176]

5. Venturini M, Bighin C, Monfardini S, Cappuzzo F, Olmeo N, Durando A, et al. Multicenter phase II study of trastuzumab in combination with epirubicin and docetaxel as first-line treatment for HER2overexpressing metastatic breast cancer. Breast cancer research and treatment. 2006; 95(1):45-53. [PubMed: 16267615]

6. Guglin M, Hartlage G, Reynolds C, Chen R, Patel V. Trastuzumab-induced cardiomyopathy: not as benign as it looks? A retrospective study. Journal of cardiac failure. 2009; 15(8):651-7. [PubMed: 19786253]

7. Cardinale D, Colombo A, Sandri MT, Lamantia G, Colombo N, Civelli M, et al. Prevention of highdose chemotherapy-induced cardiotoxicity in high-risk patients by angiotensin-converting enzyme inhibition. Circulation. 2006; 114(23):2474-81. [PubMed: 17101852]

8. Kalay N, Basar E, Ozdogru I, Er O, Cetinkaya Y, Dogan A, et al. Protective effects of carvedilol against anthracycline-induced cardiomyopathy. Journal of the American College of Cardiology. 2006; 48(11):2258-62. [PubMed: 17161256]

9. Guglin M, Cutro R, Mishkin JD. Trastuzumab-induced cardiomyopathy. Journal of cardiac failure. 2008; 14(5):437-44. [PubMed: 18514938]

10. Piccart-Gebhart MJ, Procter M, Leyland-Jones B, Goldhirsch A, Untch M, Smith I, et al. Trastuzumab after adjuvant chemotherapy in HER2-positive breast cancer. The New England journal of medicine. 2005; 353(16):1659-72. [PubMed: 16236737]

11. Leyland-Jones B, Gelmon K, Ayoub JP, Arnold A, Verma S, Dias R, et al. Pharmacokinetics, safety, and efficacy of trastuzumab administered every three weeks in combination with paclitaxel. Journal of clinical oncology: official journal of the American Society of Clinical Oncology. 2003; 21(21):3965-71. [PubMed: 14507946]

12. Ewer MS, O'Shaughnessy JA. Cardiac Toxicity of Trastuzumab-Related Regimens in HER2Overexpressing Breast Cancer. Clinical breast cancer. 2007; 7(8):600-7. [PubMed: 17592672]

13. Bengala C, Zamagni C, Pedrazzoli P, Matteucci P, Ballestrero A, Da Prada G, et al. Cardiac toxicity of trastuzumab in metastatic breast cancer patients previously treated with high-dose 
chemotherapy: a retrospective study. British journal of cancer. 2006; 94(7):1016-20. [PubMed: 16570045]

14. Bhimaraj ASS, Johnson S, Kelly R, Lad T. Trastuzumab Mediated Cardiac Dysfunction in the Clinical Setting. Journal of Cardiac Failure. 2008:S82.

15. Guarneri V, Lenihan DJ, Valero V, Durand JB, Broglio K, Hess KR, et al. Long-term cardiac tolerability of trastuzumab in metastatic breast cancer: the M.D. Anderson Cancer Center experience Journal of clinical oncology: official journal of the American Society of Clinical Oncology. 2006; 24(25):4107-15. [PubMed: 16908934]

16. Ewer MS, Vooletich MT, Durand JB, Woods ML, Davis JR, Valero V, et al. Reversibility of trastuzumab-related cardiotoxicity: new insights based on clinical course and response to medical treatment. Journal of clinical oncology: official journal of the American Society of Clinical Oncology. 2005; 23(31):7820-6. [PubMed: 16258084]

17. Seidman A, Hudis C, Pierri MK, Shak S, Paton V, Ashby M, et al. Cardiac dysfunction in the trastuzumab clinical trials experience. Journal of clinical oncology: official journal of the American Society of Clinical Oncology. 2002; 20(5):1215-21. [PubMed: 11870163]

18. Pituskin E, Mackey JR, Koshman S, Jassal D, Pitz M, Haykowsky MJ, et al. Multidisciplinary Approach to Novel Therapies in Cardio-Oncology Research (MANTICORE 101-Breast): A Randomized Trial for the Prevention of Trastuzumab-Associated Cardiotoxicity. Journal of clinical oncology: official journal of the American Society of Clinical Oncology. 2016 JCO2016687830.

19. Boekhout AH, Gietema JA, Milojkovic Kerklaan B, van Werkhoven ED, Altena R, Honkoop A, et al. Angiotensin II-Receptor Inhibition With Candesartan to Prevent Trastuzumab-Related Cardiotoxic Effects in Patients With Early Breast Cancer: A Randomized Clinical Trial. JAMA oncology. 2016; 2(8):1030-7. [PubMed: 27348762]

20. Burstein HJ, Kuter I, Campos SM, Gelman RS, Tribou L, Parker LM, et al. Clinical activity of trastuzumab and vinorelbine in women with HER2-overexpressing metastatic breast cancer. Journal of clinical oncology: official journal of the American Society of Clinical Oncology. 2001; 19(10):2722-30. [PubMed: 11352965]

21. Burstein HJ, Harris LN, Marcom PK, Lambert-Falls R, Havlin K, Overmoyer B, et al. Trastuzumab and vinorelbine as first-line therapy for HER2-overexpressing metastatic breast cancer: multicenter phase II trial with clinical outcomes, analysis of serum tumor markers as predictive factors, and cardiac surveillance algorithm. Journal of clinical oncology: official journal of the American Society of Clinical Oncology. 2003; 21(15):2889-95. [PubMed: 12885806]

22. Tan-Chiu E, Yothers G, Romond E, Geyer CE Jr, Ewer M, Keefe D, et al. Assessment of cardiac dysfunction in a randomized trial comparing doxorubicin and cyclophosphamide followed by paclitaxel, with or without trastuzumab as adjuvant therapy in node-positive, human epidermal growth factor receptor 2-overexpressing breast cancer: NSABP B-31. Journal of clinical oncology: official journal of the American Society of Clinical Oncology. 2005; 23(31):7811-9. [PubMed: $16258083]$ 


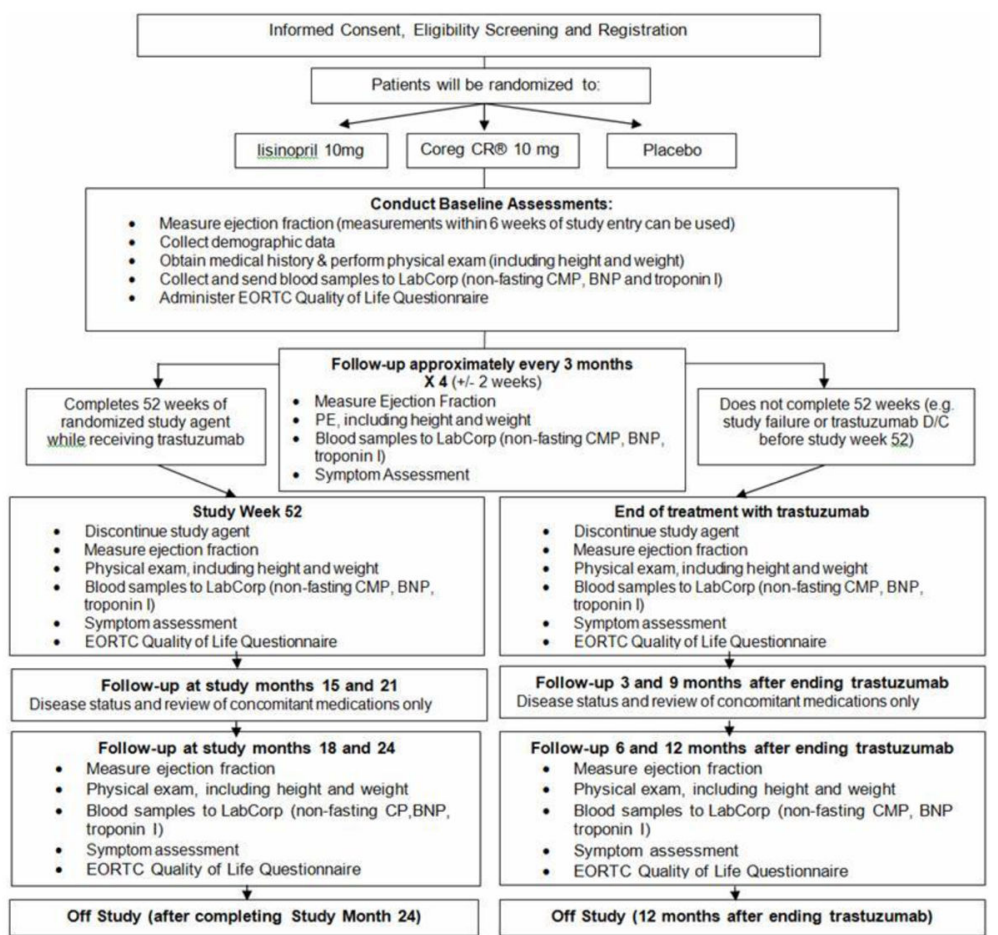

Figure 1.

SCUSF 0806 Flowchart 
Table 1

Definitions of TZB-induced cardiotoxicity at the time of designing the current clinical trial

\begin{tabular}{|c|c|c|c|c|}
\hline Author & Year & $\mathbf{N}$ & Setting & Definition of cardiotoxicity \\
\hline Burstein 20 & 2001 & 40 & Metastatic breast cancer & $\mathrm{LVEF}<50 \%$ or decrease by $15 \%$ \\
\hline Leyland-Jones ${ }^{11}$ & 2003 & 32 & Metastatic breast cancer & LVEF $<40 \%$ or decrease by $>15 \%$ \\
\hline Romond $^{3}$ & 2005 & 1159 & Adjuvant & $\mathrm{LVEF}<55 \%$ or decrease by $>16 \%$ \\
\hline Burstein $^{21}$ & 2003 & 54 & Metastatic breast cancer & $\mathrm{EF}<40 \%$ \\
\hline Bengala 13 & 2006 & 53 & mixed & $\mathrm{EF}<50 \%$ \\
\hline Tan-Chiu ${ }^{22}$ & 2005 & 850 & Adjuvant & EF decrease by $10 \%$ to $<55 \%$ \\
\hline Piccart-Gebhart ${ }^{10}$ & 2005 & 1677 & Adjuvant & $\mathrm{EF}<50 \%$ or decrease by $10 \%$ \\
\hline Venturini ${ }^{5}$ & 2006 & 45 & Metastatic breast cancer & $\mathrm{EF}<45 \%$ or decrease by $20 \%$ \\
\hline Guarneri ${ }^{15}$ & 2006 & 173 & Metastatic breast cancer & $\mathrm{EF}<50 \%$ or decrease by $20 \%$ \\
\hline
\end{tabular}

